\title{
The Sun at high resolution: first results from the SUNRISE mission
}

\author{
S. K. Solanki ${ }^{1,8}$, P. Barthol ${ }^{1}$, S. Danilovic ${ }^{1}$, A. Feller ${ }^{1}$, A. Gandorfer ${ }^{1}$, \\ J. Hirzberger ${ }^{1}$, A. Lagg ${ }^{1}$, T. L. Riethmüller ${ }^{1}$, M. Schüssler ${ }^{1}$,
} T. Wiegelmann ${ }^{1}$, J. A. Bonet ${ }^{2}$, V. Martínez Pillet ${ }^{2}$, E. Khomenko ${ }^{2}$, J. C. del Toro Iniesta ${ }^{3}$, V. Domingo ${ }^{4}$, J. Palacios ${ }^{4}$, M. Knölker ${ }^{5}$, N. Bello González ${ }^{6}$, J. M. Borrero ${ }^{6}$, T. Berkefeld ${ }^{6}$, M. Franz ${ }^{6}$, M. Roth $^{6}$, W. Schmidt ${ }^{6}$, O. Steiner ${ }^{6}$ and A. M. Title ${ }^{7}$

${ }^{1}$ Max-Planck-Institut für Sonnensystemforschung, Max-Planck-Str. 2, 37191 Katlenburg-Lindau, Germany; email: solanki@mps.mpg.de

${ }^{2}$ Instituto de Astrofísica de Canarias, C/Via Láctea s/n, 38200 La Laguna, Tenerife, Spain.

${ }^{3}$ Instituto de Astrofísica de Andalucía (CSIC), Apdo. de Correos 3004, E-18080, Granada, Spain

${ }^{4}$ Grupo de Astronomía y Ciencias del Espacio (Univ. de Valencia), E-46980, Paterna, Valencia, Spain

${ }^{5}$ High Altitude Observatory, National Center for Atmospheric Research, P.O. Box 3000, Boulder CO 80307-3000, USA. †

${ }^{6}$ Kiepenheuer-Institut für Sonnenphysik, Schöneckstr. 6, 79104 Freiburg, Germany.

${ }^{7}$ Lockheed-Martin Solar and Astrophysical Lab., Palo Alto, USA

${ }^{8}$ School of Space Research, Kyung Hee University, Yongin, Gyeonggi, 446-701, Korea

\begin{abstract}
The SUNRISE balloon-borne solar observatory consists of a $1 \mathrm{~m}$ aperture Gregory telescope, a UV filter imager, an imaging vector polarimeter, an image stabilization system and further infrastructure. The first science flight of SUNRISE yielded high-quality data that reveal the structure, dynamics and evolution of solar convection, oscillations and magnetic fields at a resolution of around $100 \mathrm{~km}$ in the quiet Sun. Here we describe very briefly the mission and the first results obtained from the SunRISE data, which include a number of discoveries.
\end{abstract}

Keywords. Sunrise, telescope, quiet sun, instrumentation

\section{Introduction}

SUNRISE is the latest in a long line of solar telescopes carried by stratospheric balloons. It combines high spatial resolution with sensitivity to ultraviolet radiation. At $1 \mathrm{~m}$ diameter, it is the largest solar telescope so far to leave the ground. It is equipped with state-of-the-art post-focus instruments, including a UV imager and a filter-based vector magnetograph.

The magnetic field in the solar photosphere shows a very complex and diverse structure. Concentrations of magnetic field with kilo-Gauss strength appear in a broad range of sizes reaching down to small flux concentrations on scales of $100 \mathrm{~km}$ or below. SUNRISE aims to determine the true size and brightness distribution of the concentrated magnetic features by spatially resolving them, as well as providing greatly improved properties of the internetwork fields. SunRISE also aims to probe the convection in the solar photosphere, as well as the often complex effects of the interaction of the magnetic field with the turbulent convection.

$\dagger \mathrm{HAO} / \mathrm{NCAR}$ is sponsored by the NSF 


\section{Instrumentation and Mission}

The SunRISE stratospheric balloon-borne observatory is composed of a telescope, two post-focus science instruments (called SuFI and IMaX, see below), an Image Stabilization and Light Distribution (ISLiD) unit, and a Correlating Wave-front Sensor (CWS), all supported by a gondola, which possesses pointing capability.

The telescope is a Gregory-type reflector with $1 \mathrm{~m}$ clear aperture and an effective focal length of close to $25 \mathrm{~m}$. A heat-rejection wedge at the prime focus reflects $99 \%$ of the light from the solar disk off to the side, reducing the heat load on the post-focus instruments to approximately $10 \mathrm{~W}$. The post-focus instrumentation rests on top of the telescope. More details on the telescope, gondola and mission are given by Barthol et al. (2010), while the CWS is described in detail by Berkefeld et al. (2010).

The SunRISE Filter Imager (SuFI) provides images in 5 narrow and medium bands at violet and near ultraviolet wavelengths between roughly 200 and $400 \mathrm{~nm}$. The highest cadence that can be achieved is an image every $2 \mathrm{~s}$. In order to overcome aberrations, a phase-diversity technique (e.g. Paxman et al. 1992) is employed, also by the other science instrument, IMaX. A description of SuFI can by found in Gandorfer et al. (2010).

The Imaging Magnetograph eXperiment (IMaX) operates in the Zeeman $g=3 \mathrm{Fe}$ I $525.02 \mathrm{~nm}$ line. Images in polarized light covering $50 \times 50 \operatorname{arcsec}^{2}$ are recorded at a spectral resolution of $85 \mathrm{~m} \AA$, normally at 4 wavelengths within the spectral line and 1 in the nearby continuum. The full Stokes vector in these 5 wavelengths at a noise level of $10^{-3}$ is obtained in $30 \mathrm{sec}$, which is the typical cadence for most of the observations. A dual-beam approach is taken, with 2 synchronized $1 \mathrm{k} \times 1 \mathrm{k}$ CCD cameras. Detailed information on IMaX is provided by Martínez Pillet et al. (2010).

SUNRISE was flown on a zero-pressure stratospheric long-duration balloon launched on June 8, 2009 from ESRANGE near Kiruna in northern Sweden. It then floated westwards at a mean cruise altitude of $36 \mathrm{~km}$ and landed on Somerset island (northern Canada), suspended on a parachute, on June 13, 2009. At float altitudes, virtually seeing-free observations were possible all the time (since the payload was above more than $99 \%$ of the Earth's atmosphere).

The loss of high-speed telemetry relatively soon after reaching float altitude (due to the failure of a rented commercial telemetry system), meant that no full images could be downloaded during the entire mission. Consequently instrument commissioning and operations had to be carried out practically blindly. Nonetheless, the achieved spatial resolution was sufficiently high to resolve both, small-scale magnetic and convective features (Lagg et al. 2010, Khomenko et al. 2010).

\section{First results}

The Sun was extremely quiet during the entire flight of SunRISE, so that almost all of the gathered data correspond to internetwork regions with occasional network elements. An overview of the data and first results is given by Solanki et al. (2010).

Images of the quiet Sun at disk centre in all $5 \mathrm{SuFI}$ wavelengths are shown in Fig. 1a, whose grey scale is saturated at $\langle I\rangle \pm 3 \sigma$ for each wavelength in order to allow a better intercomparison of the granulation, at the cost of overexposing the bright points. The brightness scale (see the gray-scale bars above the individual frames) already indicates the large rms contrasts of the imaged granulation, which reach up to $32 \%$ at $214 \mathrm{~nm}$ (cf. Hirzberger et al., 2010a, for a complete study of the rms contrasts). At a number of the observed wavelengths the contrasts can be compared with those resulting from the $3 \mathrm{D}$ radiation-MHD simulations of Vögler et al. (2005) and are found to be in good 

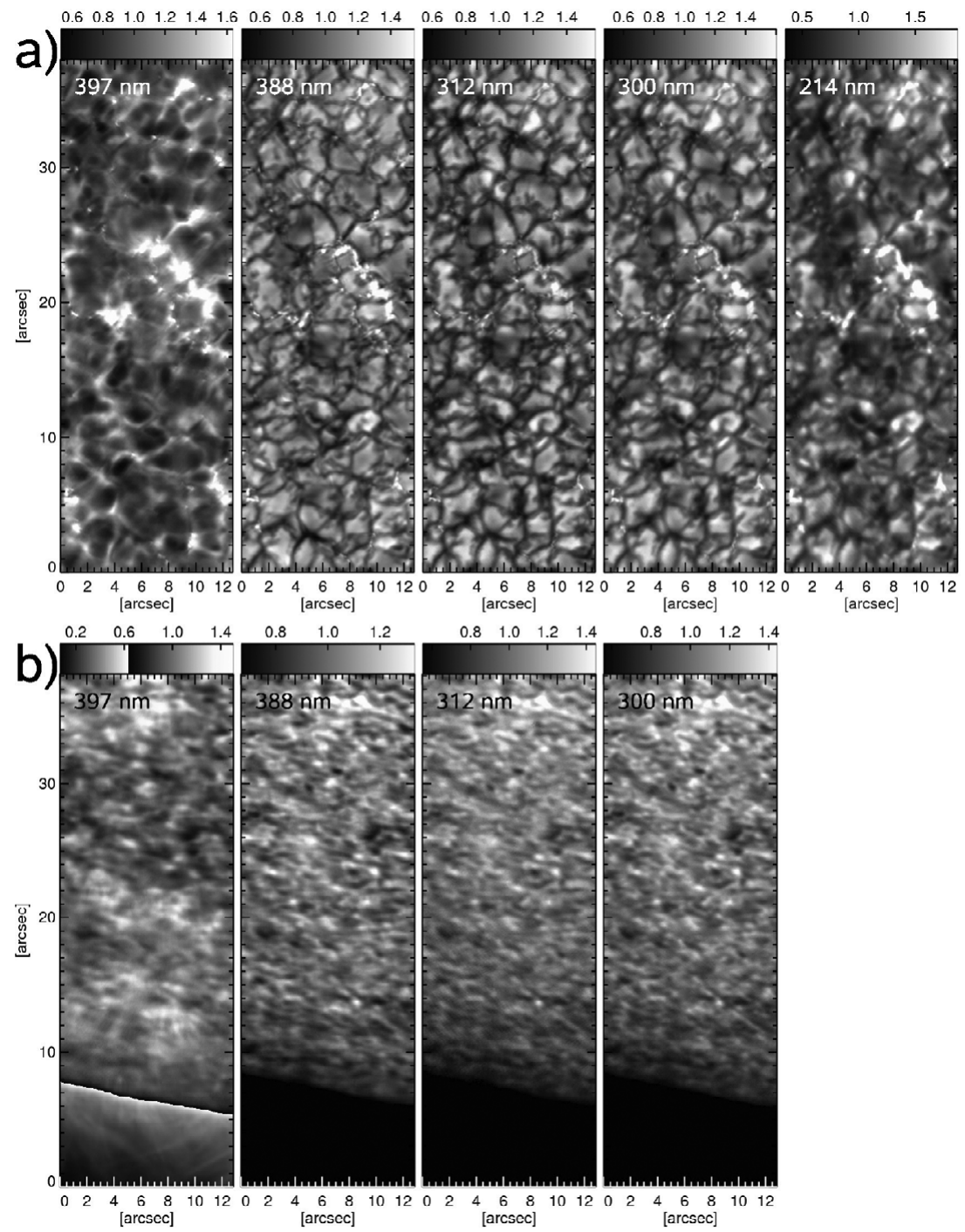

Figure 1. a) Images of a patch of quiet Sun near disk centre recorded by the SuFI instrument in wavelength bands centred on $397 \mathrm{~nm}, 388 \mathrm{~nm}, 312 \mathrm{~nm}, 300 \mathrm{~nm}$ and $214 \mathrm{~nm}$ (from left to right). The grey scale has been individually set to cover 3 times the RMS range of each image. b) Same as panel a, but at the solar limb. The Ca H image $(397 \mathrm{~nm})$ is plotted with an enhanced brightness scale for the off-limb parts. No $214 \mathrm{~nm}$ data are available at this position.

agreement. This supports both the high resolution and very low stray light of SUNRISE SuFI data.

Bright points are prominent at all wavelengths sampled by SuFI, but are particularly so at $214 \mathrm{~nm}$ (they are 2.3 times as bright as the background at this wavelength, see Riethmüller et al., 2010), making them brighter than at any other wavelength studied so far.

Figure 2 shows a snapshot of IMaX data products. Stokes- $V$ movies (see e.g. Solanki et al. 2010) reveal how dynamic the quiet Sun magnetic field is, with the weaker magnetic features, i.e., those in the internetwork, being particularly dynamic. 

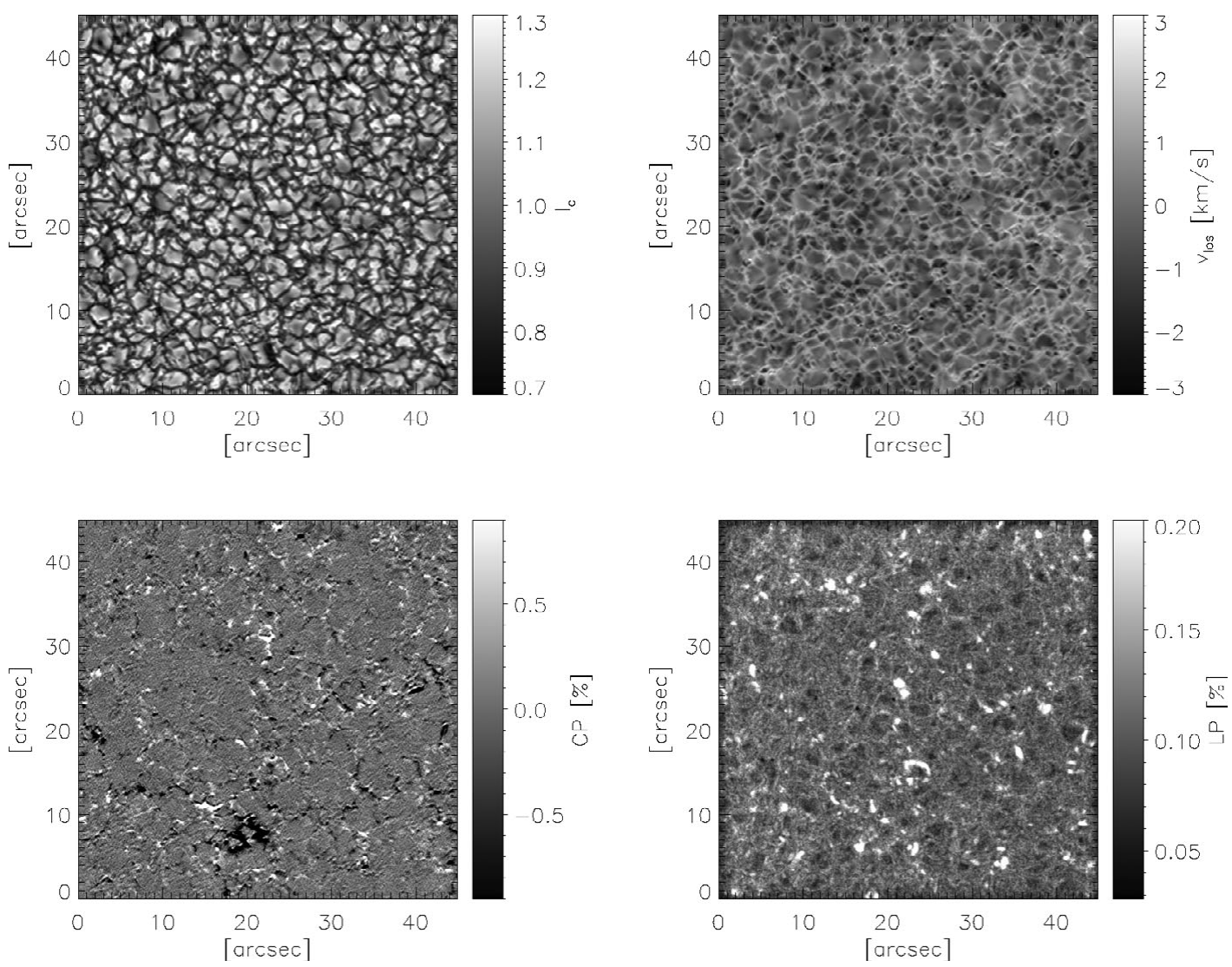

Figure 2. IMaX data. Clockwise from upper left: continuum intensity at $5250.4 \AA$, line-of-sight velocity, net linear polarization $L_{s}$ averaged over the line, $V_{s}$, the line-averaged Stokes $V$ obtained from Fe I $5250.2 \AA$. All images are based on phase-diversity reconstructed data except for the linear polarization image (reconstruction increases the noise, so that a number of significant $L_{s}$ patches in the unreconstructed image are no longer sufficiently above the noise in the reconstructed data).

An investigation of the properties of small concentrations of strong magnetic fields in the quiet Sun showed that these have finally been resolved by IMaX on SUNRISE (Lagg et al. 2010). An inversion technique applied to retrieve the temperature stratification and the field strength could reproduce the observations well with a one-component, fully-magnetized atmosphere with a field strength exceeding $1 \mathrm{kG}$ and a significantly enhanced temperature in the mid to upper photosphere compared to its surroundings. This is consistent with semi-empirical flux tube models describing magnetic elements. Consequently, it can be concluded that the SUNRISE measurements resolve the observed quiet Sun flux tubes. This result suggests that the SunRISE data will allow further properties of these basic building blocks of the photospheric magnetic field to be determined rather directly.

SUNRISE observations show that the occurrence rate of patches of significant linear polarization signal (sensitive to the transverse component of photospheric magnetic field) is 1-2 orders of magnitude larger than values reported by previous studies (Danilovic et al. 2010). These features appear preferentially at granule boundaries with most of them being caught in downflow lanes at some point in their evolution. Only a small percentage are 
entirely and constantly embedded in upflows (16\%) or downflows (8\%). For the latter, the usual interpretation in terms of magnetic flux emerging from below cannot hold, so that they must have another source.

Borrero et al. (2010) discovered that some of the patches of horizontal magnetic field in the internetwork are associated with supersonic upflows of magnetized gas. An interpretation in terms of localized jets and heating due to magnetic reconnection in photopsheric layers between the emerging and previously present magnetic flux seems plausible.

An analysis by Bello Gonzalez et al. (2010) of high-resolution spectropolarimetric data obtained by IMaX on SUNRISE provides a total energy flux of approximately 6400-7700 $\mathrm{W} \mathrm{m}{ }^{-2}$ at a height of $250 \mathrm{~km}$ carried by waves with a period shorter than $3 \mathrm{~min}$. This is more than twice the energy flux found in any previous work and lies within a factor of 2 of the energy flux needed to balance radiative losses from the chromosphere according to Anderson \& Athay (1989). This result revives interest in acoustic waves for transporting energy to the chromosphere. An interesting conclusion is that the "missing" acoustic flux is hidden mainly at small spatial scales and not so much at high frequencies, where it has been assumed to lie in the past.

SUNRISE data have revealed vortical motion at small scales, with vorticity in both the horizontal and the vertical direction. Flows with vorticity in the vertical direction are seen by following small magnetic field patches (Bonet et al. 2010), while (smaller) horizontally directed vortex tubes have been discovered at the edges of granules by comparing time series of continuum images with 3D hydrodynamic simulations by Steiner et al. (2010).

The high resolution of the data is also indicated by the work of Khomenko et al. (2010), who show that the intergranular lanes, associated with broadened spectral lines, are bordered by narrow stripes of narrow spectral lines. These stripes, visible only at the high resolution achieved by SunRISE, are located where the granular flows bend from upto downflows, as comparisons with 3D hydrodynamic simulations show.

High degree $p$-modes are studied by Roth et al. (2010), who find that the power in waves with degree $l>1000$ is enhanced over granules shortly before these start to split or explode, suggesting a connection between granule evolution and excitation of $p$-modes.

Finally, the magnetic field measured by SUNRISE in the photosphere has been extrapolated into the upper solar atmosphere by Wiegelmann et al. (2010). A statistical study of the connectivities of the extrapolated field shows that almost all of the magnetic field lines reaching the chromosphere or higher connect network with internetwork patches in the photosphere. Since the internetwork field is extremely dynamic and short-lived, this implies that the magnetic field in the upper atmosphere must also be changing very rapidly.

\section{Conclusion}

The SunRISE observatory has provided high-quality, high-resolution images, Dopplergrams and vector magnetograms at different positions on the solar disk. The extremely low solar activity level at the time of the science flight of SunRISE means that these data mainly enable new insights into the quiet Sun.

An initial analysis of these data has already led to new insights into the magnetism, convection, oscillations and waves in the quiet Sun. Given the richness and quality of the data and the fact that so far only a small fraction of them have been analyzed, we expect many more exciting results to follow. A flight of SUNRISE during a period of higher solar activity is greatly to be welcomed. 


\section{Acknowledgements}

The German contribution to SUNRISE is funded by the Bundesministerium für Wirtschaft und Technologie through Deutsches Zentrum für Luft- und Raumfahrt e.V. (DLR), Grant No. 50 OU 0401, and by the Innovationsfond of the President of the Max Planck Society (MPG). The Spanish contribution has been funded by the Spanish MICINN under projects ESP2006-13030-C06 and AYA2009-14105-C06 (including European FEDER funds). The HAO contribution was partly funded through NASA grant number NNX08AH38G. This work has been partially supported by the WCU grant No. R3110016 funded by the Korean Ministry of Education, Science \& Technology.

\section{References}

Anderson, L. S. \& Athay, R. G. 1989, Astrophys. J., 336, 1089

Barthol, P., Gandorfer, A., Solanki, S. K., et al. 2010, Solar Phys., 268, 1

Bello González, N., Franz, M., Martínez Pillet, V., et al. 2010, Astrophys. J., 723, L134

Berkefeld, T., Schmidt, W., Soltau, D., et al. 2010, Solar Phys., 723, L139

Bonet, J. A., Márquez, I., Sánchez Almeida, J., et al. 2010, Astrophys. J., 723, L134

Borrero, J. M., Martínez Pillet, V., Schlichenmaier, R., et al. Astrophys. J., 723, L144

Danilovic, S., Beeck, B., Pietarila, A., et al. 2010a, Astrophys. J., 723, L149

Gandorfer, A., Grauf, B., Barthol, P., et al. 2010, Solar Phys., 268, 14

Hirzberger, J., Feller, A., Riethmüller, et al. 2010a, Astrophys. J., 723, L254

Khomenko, E., Martínez Pillet, V., \& Solanki, S. K., 2010, Astrophys. J., 723, L159

Lagg, A., Solanki, S. K., Riethmüller, T. L., et al. 2010, Astrophys. J., 723, L164

Martínez Pillet, V., del Toro Iniesta, J. C., Álvarez-Herrero, A., et al. 2010, Solar Phys., in press

Paxman, R. G. and Schulz, T. J., \& Fienup, J. R. 1992, J. Optical Soc. America A, 9, 1072

Riethmüller, T. L., Solanki, S. K., Martínez Pillet, V., et al. 2010, Astrophys. J., 723, L127

Roth, M., Franz, M., Bello González, N., et al. 2010, Astrophys. J., 723, L175

Solanki, S. K., Barthol, P., Danilovic, S., et al. 2010, Astrophys. J., 723, L127

Steiner, O., Franz, M., Bello González, N., et al. 2010, Astrophys. J., 723, L164

Vögler, A., Shelyag, S., Schüssler, M., Cattaneo, F., Emonet, T., \& Linde, T. 2005, Astron. Astrophys, 429, 335

Wiegelmann, T., Solanki, S. K., Borrero, J. M., et al. 2010, Astrophys. J., 723, L185

\section{Discussion}

Georgoulis: What is the achieved spatial resolution, the magnetic filling factor, and the noise level of the data?

SolankI: The Noise level is a couple of times ten to the minus three if you take a single magnetogram. There are of course techniques of averaging magnetograms and reducing the noise level and so on, and that's something we're working on. These are just some of the results.

KLIMCHUK: So the Kilogauss really are existing in tubelike structures and not these thin stretched-out lane-type features?

SolAnkI: The noise level is very quiet region, yes; but that's because you have very little flux. So also in the simulations, if you take an image D simulation with a very low amount of magnetic flux, you will tend to get little point-like flux tubes. As you increase the amount of magnetic flux, it will not just stay there at the intersection of intergranules lanes, but it will start filling up these lanes, and then you will start getting these more elongated structures. 
So we want refly again definitely and hopefully at a time when the sun is more active and you won't see just quiet features and hopefully also see these sheet-like structures.

Kitiashvili: Do you want to repeat during the solar cycle maxima?

SolAnki: Yes, we definitely plan to do that. So if all goes well, we would like to refly again in 2012 which at the moment is thought to be a time where the sun will be hopefully more active than in 2009. We still haven't got 100 percent of the funding yet, but we are relatively confident we will be able to do that. 